\title{
Carreras delictivas juveniles y tratamiento ${ }^{1}$
}

\section{Ana Martínez-Catena*}

<a.martinez.catena@ub.edu>

\author{
Santiago Redondo Illescas* \\ * Dpto. de Personalidad, Evaluación y Tratamiento Psicológico. \\ Facultad de Psicología, Universidad de Barcelona
}

Ibilbide kriminalen inguruko ikerkuntzak nabarmendu izan du gazte-delinkuentzia prebentzio konplexuko fenomenoa dela duen faktore anitzeko etiologiagatik. Ondorengo testu honetan azaltzen dira joandako urteotan ikerkuntzak jokabide delitugilea gertatzeko aukera gehiago dituzten nabarmendutako arrisku-faktoreak. Halaber, berrikusten dira nazioartean gehien erabili eta eraginkortasun nabarmenena erakutsi duten eskuartze terapeutikoek oinarri dituzten ikuspegi teorikoak. Horretaz gain, ikuspegi aplikatu bati jarraituz deskribatzen dira espainiar komunitate batzuetan adingabekoak gizarteratzeko burutu diren ekimen eta gizarte-hezkuntzako programa garrantzitsuenak. Azkenik, aurrekoaren hariari jarraikiz, azterlan honetan erlazionatzen dira Espainian garatutako aplikazio praktikoak berrikusitako ibilbide kriminal, arrisku-faktore eta eraginkortasun terapeutikoaren inguruko ezaguerarekin.

\section{HITZ-GAKOAK:}

Gazte-delinkuentzia, arrisku-faktoreak, delitugileen tratamendua, gizarte-hezkuntzako eskuartzea, eraginkortasuna.
La investigación sobre carreras criminales ha destacado que la delincuencia juvenil es un fenómeno de compleja prevención, debido a su etiología multifactorial. En el siguiente trabajo, se exponen los principales factores de riesgo asociados a una mayor probabilidad de conducta delictiva que la investigación ha identificado a lo largo de los últimos años. Así mismo, se revisan las perspectivas teóricas sobre las que se fundamentan las intervenciones terapéuticas más utilizadas internacionalmente y que han demostrado una mayor eficacia. Además, desde un punto de vista aplicado, se describen las actuaciones y programas socioeducativos más importantes que se dirigen a la reintegración social de los menores aplicados en las diversas comunidades españolas. Finalmente, en coherencia con lo anterior, en este trabajo se pone en relación las aplicaciones prácticas desarrolladas en España con los conocimientos revisados sobre carreras criminales, factores de riesgo y eficacia terapéutica.

\section{PALABRAS CLAVE:}

Delincuencia juvenil, factores de riesgo, tratamiento de delincuentes, intervención socioeducativa, eficacia.

\footnotetext{
${ }^{1}$ Este trabajo se fundamenta en los resultados de investigación obtenidos en el marco del proyecto de investigación titulado "Factores de éxito asociados a los programas de intervención con menores infractores”, promovido y financiado por la Dirección General de Política Social, de las Familias y de la Infancia (Ministerio de Sanidad y Política Social) y del Proyecto Coordinado I+D (SEJ2005- 09170-C04-01/PSI) titulado "Delincuencia violenta: programas de prevención, tratamiento y predicción”.
} 


\section{Presentación}

La delincuencia juvenil constituye una preocupación constante para las sociedades y sus Gobiernos. En todas las generaciones de jóvenes, muchos de ellos cometen diversas infracciones, generalmente leves, y un grupo muy minoritario devienen delincuentes persistentes. Por ello, la delincuencia juvenil constituye también un objetivo preferente del estudio científico de la criminalidad. En este trabajo se atenderá, en primer lugar, a describir la magnitud y prevalencia de la delincuencia juvenil, así como sus categorías infractoras principales; a continuación, se revisará la investigación actual sobre carreras delictivas y factores de riesgo para la conducta antisocial, a la vez que las conexiones existentes entre la delincuencia juvenil y la delincuencia adulta; $y$, por último, se presentarán algunos programas e intervenciones educativas con jóvenes y sus resultados más importantes. Todo ello se hará tomando en cuenta tanto la investigación internacional como los más recientes resultados existentes en España².

\section{Magnitud del fenómeno de la delincuencia juvenil}

Investigaciones internacionales recientes sitúan la prevalencia delictiva, o proporción de jóvenes sobre el conjunto de la población juvenil de un territorio que cometen una infracción, en torno al $80 \%$ de los menores (Farrington, 2008; Howell, 2009; McMurran y Hollin, 1993). Esto significa que existe una prevalencia delictiva juvenil elevada, pero, afortunadamente, circunscrita a infracciones generalmente leves, tales como hurtos en tiendas o uso inadecuado de Internet, por ejemplo. (Howell, 2009; Redondo y Garrido, 2001). Del mismo modo, en España se ha puesto de relieve, mediante estudios de autoinforme sobre el comportamiento delictivo de los jóvenes, que alrededor de un 98,8\% de los adolescentes han cometido una conducta ilícita leve en algún momento de su vida (Rechea, 2008; Rechea et al., 1995). En concreto, Rechea (2008) encontró, en una muestra de 4.152 escolares, que las conductas antisociales más frecuentemente realizadas fueron hechos en general leves, como bajar música mediante Internet ( $66 \%$ de la muestra), consumir bebidas alcohólicas siendo menores de edad (63\%), haberse emborrachado ( $41 \%)$, consumir cannabis $(28 \%)$ y participar en peleas $(22 \%)$.

También a través de los estudios de autoinforme, tanto nacionales como internacionales, se ha indicado que la edad en que la mayoría de jóvenes manifiestan haber cometido su primera infracción legal es 13 años, mientras que la edad de máxima frecuencia infractora se sitúa en torno a los 16 y 17 años.

${ }^{2}$ A lo largo de este trabajo se utilizarán indistintamente los términos de 'menores', 'jóvenes infractores', o ‘infractores', para referirse al conjunto de jóvenes menores de 18 años que han cometido una infracción penal.
Otra conclusión general que se obtiene en este tipo de estudios es que el porcentaje de chicas infractoras es inferior al de varones en todos los comportamientos ilícitos y antisociales analizados, con la excepción de la conducta de consumo de alcohol y cannabis, y el robo en tiendas (Farrington, 1992, 2008; Rechea, 2008; Redondo y Garrido, 2013; Sampson y Laub, 2005).

Además se ha destacado el hecho de que, en un reducido grupo de jóvenes, la incidencia delictiva, es decir, la frecuencia con la que los menores cometen delitos, es bastante más elevada. Este pequeño conjunto de jóvenes, estimado en torno al $5 \%$, suele ser el responsable de la mayoría de los delitos cometidos en un territorio, especialmente de los más graves y violentos (Bechtel, Lowenkamp y Latessa, 2007; Henggeler, 1989, 2003; Loeber, Farrington y Waschbusch, 1998; Lösel, 2000). Al mismo tiempo, estos jóvenes suelen persistir y agravar su actividad delictiva, desarrollando carreras criminales estables y crónicas. Este grupo reducido de menores será con mayor probabilidad objeto de la aplicación de medidas penales juveniles (Farrington, 2008; Howell, 2009; Moffit, 1993).

El análisis de las cifras oficiales de delincuencia juvenil en España, tomando como base el total de jóvenes condenados por cometer un delito o una infracción delictiva entre 2007 y 2012, muestra una tendencia más o menos estable (con moderados altibajos, tal y como suele suceder en todos los fenómenos sociales) [Gráfico 1]. No obstante, los análisis que siguen deben ser interpretados con cautela, debido a la dificultad de encontrar datos fiables y comparables, que permitan extraer conclusiones concretas acerca de la magnitud real del fenómeno delictivo en España. Estas dificultades se ven extremadas por lo que a cifras de delincuencia juvenil se refiere, debido a motivos diversos, como la protección de datos, el cambio recurrente de las instituciones responsables de computar las cifras delictivas, el empleo de diferentes tipos de datos sobre hechos delictivos (denuncias, sentencias, condenas, medidas en ejecución) o el hecho de que algunos de estos datos no sean formalmente publicados.

En todo caso, si se analiza por tramos de edad la cifra de menores condenados en 2012, se observa que el período de 16 a 17 años es el que presenta una mayor prevalencia de condenas, con 4.758 jóvenes sentenciados (INE, 2012). Dicha cifra correspondería al $65 \%$ del total de condenas aplicadas a menores de edad en 2012 , lo que refleja, tal y como se ha mencionado anteriormente, que el período de máxima frecuencia delictiva se produce a dicha edad. No obstante, si se toman en consideración las condenas de jóvenes adultos, es decir, de sujetos de entre 18 y 20 años que son juzgados a partir del Código Penal adulto, en 2012, el número de condenas ascendió a 15.718 jóvenes (ibídem). Es decir, que la cifra de jóvenes adultos condenados es claramente superior a la globalidad de los menores sentenciados. 


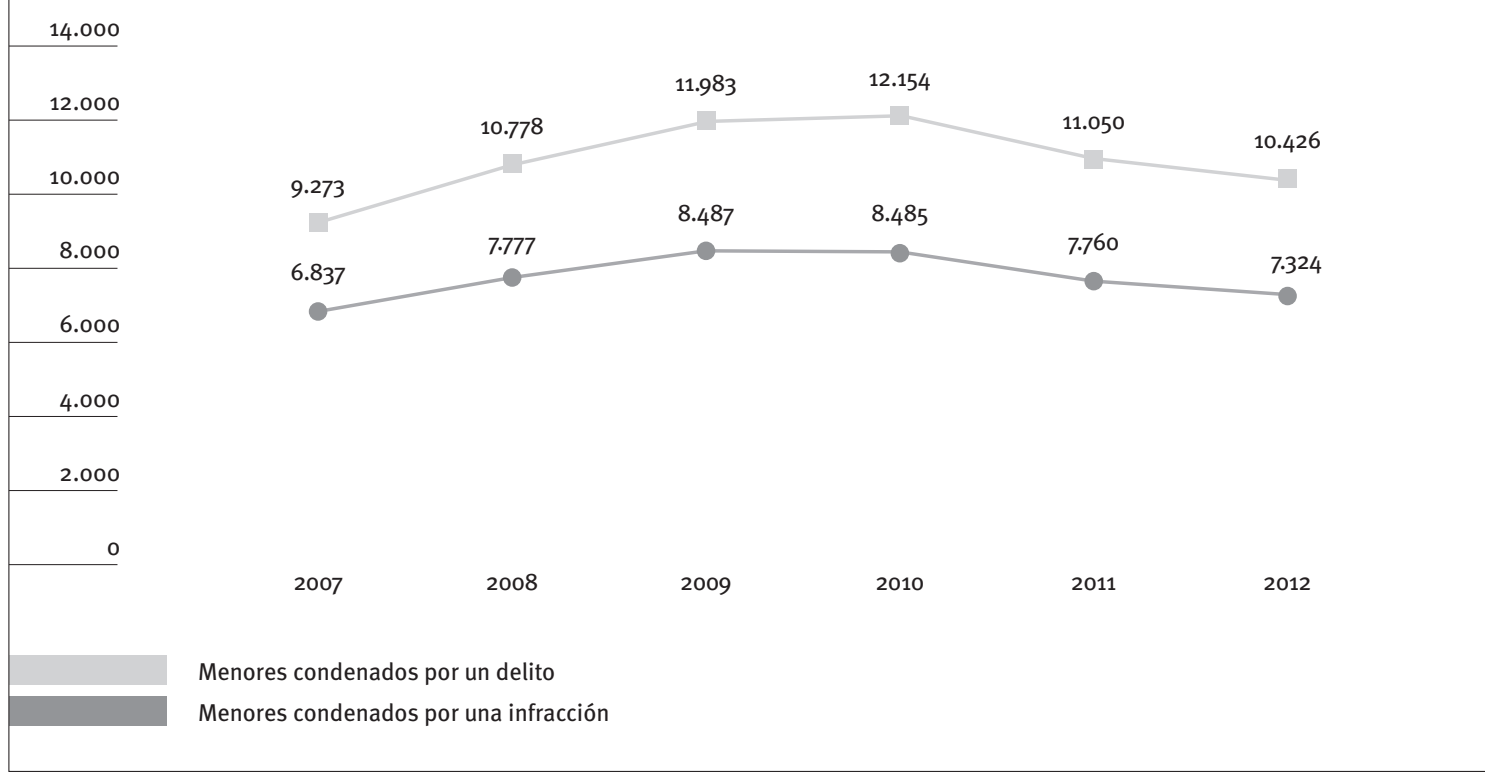

Fuente: Elaboración propia a partir de datos del Insituto Nacional de Estadística.

\subsection{Infracciones más frecuentes de los jóvenes}

Atendiendo al tipo de delito cometido por los jóvenes en los últimos años, los datos muestran que los delitos contra la propiedad son los más frecuentes (Tabla 1). En segundo lugar, se encontraría el delito de lesiones, seguido, muy por debajo, de los delitos contra la salud pública, contra la libertad sexual y, por último, del homicidio y sus distintas formas.

Tabla 1. Número de menores condenados según la tipología delictiva. España, 2007-2012

\begin{tabular}{l|c|c|c|c|c|c}
\cline { 2 - 7 } & 2007 & 2008 & 2009 & 2010 & 2011 & 2012 \\
\hline $\begin{array}{l}\text { Contra el } \\
\text { patrimonio }\end{array}$ & 4.248 & 4.654 & 4.697 & 4.768 & 4.546 & 4.167 \\
\hline Lesiones & 929 & 1.493 & 1.142 & 1.070 & 1.065 & 1.060 \\
\hline $\begin{array}{l}\text { Contra la } \\
\text { libertad } \\
\text { sexual }\end{array}$ & 109 & 152 & 148 & 167 & 117 & 140 \\
\hline $\begin{array}{l}\text { Contra la } \\
\text { salud pública }\end{array}$ & 195 & 264 & 230 & 227 & 184 & 186 \\
\hline $\begin{array}{l}\text { Homicidio y } \\
\text { sus formas }\end{array}$ & 16 & 19 & 23 & 36 & 14 & 19 \\
\hline $\begin{array}{l}\text { Total } \\
\text { condenas } \\
\text { (delitos) }\end{array}$ & 6.837 & 7.777 & 8.487 & 8.485 & 7.760 & 7.324 \\
\hline
\end{tabular}

Fuente: Elaboración propia a partir de datos tomados del Instituto Nacional de Estadística (2012).

Los delitos contra la propiedad, ya sean realizados por jóvenes o adultos, constituyen el grueso de los hechos delictivos cometidos en un territorio (a excepción del aumento reciente de los delitos contra la seguridad vial realizados por adultos). Así mismo, en comparación con otros países europeos, los delitos contra la propiedad también superan el $70 \%$ del total de las denuncias.

La mayoría de las infracciones son delitos menores, como el hurto o el robo, pero otras pueden comportar diversos grados de fuerza o violencia. En 2012, se produjeron en España 3.045 condenas de jóvenes por robo, cifra que representa el $18,82 \%$ del total de 16.172 menores condenados por delitos y otras infracciones en ese año. Además, las condenas por hurto (450 condenas de jóvenes) y robos de vehículo de motor (132 condenas) constituyen también infracciones muy frecuentes entre los delitos contra la propiedad. Como se observa en la Tabla 1, las condenas de jóvenes referidas a las infracciones contra el patrimonio aumentaron ligeramente a partir de 2008, para volver a decrecer en 2012.

Según los autoinformes de los jóvenes españoles (Rechea, 2008), el tipo de infracción contra la propiedad que cometen con mayor frecuencia es el robo (en realidad, generalmente, hurto) en tiendas (20,9\%), siguiéndole el hurto de bicicletas o ciclomotores $(3,7 \%)$, y la sustracción de objetos del interior de los vehículos (3,6\%). Por fortuna, sólo un 1,1\% de los jóvenes afirman haber realizado un tirón de bolso o cartera para quitarle algo a alguien por la fuerza, delito claramente más grave.

Los delitos contra el patrimonio persiguen claramente la obtención de gratificaciones materiales. En ciertos casos, el hurto y el robo constituyen, para algunos jóvenes, un medio de procurarse los bienes 
deseados o necesitados, especialmente en el caso de aquellos jóvenes con mayores carencias socioeconómicas. De cualquier forma, estas infracciones son una manera fácil y eficaz de obtener de modo inmediato y sin esfuerzo el objeto deseado. En función de si las consecuencias del delito han resultado gratificantes para el sujeto, el comportamiento delictivo llegará a consolidarse en su repertorio conductual.

El delito de lesiones sería el segundo tipo de infracción cometida con mayor frecuencia por los jóvenes, suponiendo casi un 9,2\% del total de sus condenas. Muchas de estas agresiones entre adolescentes suelen ser el resultado de recíprocas provocaciones entre individuos. Así mismo, esta violencia puede ejercerse de forma grupal, en defensa del que consideran su propio territorio en el barrio, en protección de sus señas de identidad, o por el logro de su primacía sobre un grupo rival. También puede producirse violencia xenófoba contra extranjeros e inmigrantes, o contra grupos sociales específicos, como homosexuales, travestís, indigentes u otros.

Por otro lado, en una proporción mucho menor a la que representan las anteriores infracciones, se encontrarían delitos más violentos, como las agresiones sexuales y el homicidio. En relación a los delitos de carácter sexual, en 2012 se condenó a 140 jóvenes, aproximadamente un 0,5\% de la población adulta imputada por estos delitos (que ascendió a 2.457 personas). Además, ese mismo año se condenó a 19 jóvenes por delitos de homicidio, lo que supone en torno a un $0,1 \%$ del total de las condenas de jóvenes.

Por último, se encuentran las infracciones relacionadas con la producción, distribución y consumo de drogas. En España, durante 2012 se dictaron un total de 186 condenas a menores por delitos contra la salud pública, frente a los 16.766 adultos condenados por esta modalidad delictiva. Esta cifra de condenas hace referencia, en exclusiva, a los delitos directamente relacionados con las propias drogas, es decir, con conductas de fabricación, posesión, venta o consumo en la vía pública. Sin embargo, a estas condenas cabría sumar todos aquellos delitos distintos, pero vinculados a la distribución de drogas, como robos, agresiones y extorsiones relacionadas con el tráfico de drogas. En este sentido, internacionalmente se estima que estos delitos conectados de forma secundaria con el consumo de sustancias tóxicas representarían entre un $40 \%$ y un $60 \%$ de los que se cometen en un territorio (especialmente, de los hechos violentos).

Tras observar la prevalencia de los diferentes tipos delictivos cometidos preferentemente por los jóvenes, se plantea otra cuestión de alta relevancia para el análisis de las carreras criminales juveniles: la dicotomía entre especialización y versatilidad delictiva. Es decir, si los jóvenes que cometen delitos con cierta frecuencia lo hacen preferentemente de un modo especializado (centrándose en un solo tipo delictivo), o más bien de manera generalista o versátil (o sea, que pueden cometer diferentes tipos de delitos). Aunque, por el momento, no existe una respuesta categórica a esta disyuntiva, lo más cierto es que la mayoría de los jóvenes infractores propenden a ser más versátiles que especializados, aunque en algunos infractores persistentes se produce una cierta especialización.

\section{Investigación básica sobre carreras delictivas juveniles}

\subsection{Factores de riesgo y de protección para la delincuencia}

A lo largo de las últimas décadas, gracias a los estudios longitudinales, se han descrito múltiples factores de riesgo asociados a una mayor probabilidad de comportamiento delictivo (Bergman, 2009; Bergman y Andershed, 2009; Farrington, Ttofi y Coid, 2009; Lee, Beaver y Wright, 2009; Nilson y Estrada, 2009; Remschmidt y Walter, 2009; Sanercki, 1985, 2009; Stenberg et al., 2007). También se han detallado numerosos factores de protección, los cuales se asocian a una menor probabilidad de conducta antisocial. En muchos casos, los factores de protección aparecerían como polo opuesto a los anteriores riesgos, amortiguando su influjo sobre el individuo.

Tradicionalmente, los correlatos de riesgo han sido agrupados en distintas fuentes o categorías, en función de su procedencia o ámbito de incidencia. Una clasificación reciente, propuesta por el modelo del triple riesgo delictivo (TRD) [Redondo, 2008a], sugiere la diferenciación entre factores de riesgo de tipo personal, factores de riesgo en el apoyo social recibido y factores de riesgo situacionales.

Seguidamente se presenta una revisión de los correlatos o factores de riesgo con mayor confirmación empírica (Cuadro 1). Como se puede observar, entre los personales, se incluyen factores relacionados con aspectos genéticos, de personalidad, conductuales, relativos a los modos de pensar y sentir de los jóvenes, y con ciertos déficits intelectivos y de aprendizaje. Los factores de riesgo sociales conciernen a las posibles carencias de los jóvenes en cuatro áreas en las que suele transcurrir su vida diaria: el barrio, la familia, la escuela y los amigos. Y finalmente, en el grupo de factores de tipo situacional se recogen aquellos elementos ambientales que favorecen la aparición de nuevas oportunidades para la comisión de delitos.

Diversos modelos teóricos han conectado los factores de riesgo para el delito que presenta un sujeto con la intervención o tratamiento rehabilitador. Un principio bien establecido es que, mediante la eliminación o la disminución de la fuerza con la que inciden los factores de riesgo, la probabilidad de cometer nuevos delitos se reducirá. Por ello, otra distinción relevante, por sus implicaciones en el tratamiento de los delincuentes, diferencia los citados riesgos entre factores estáticos y dinámicos. Los primeros 


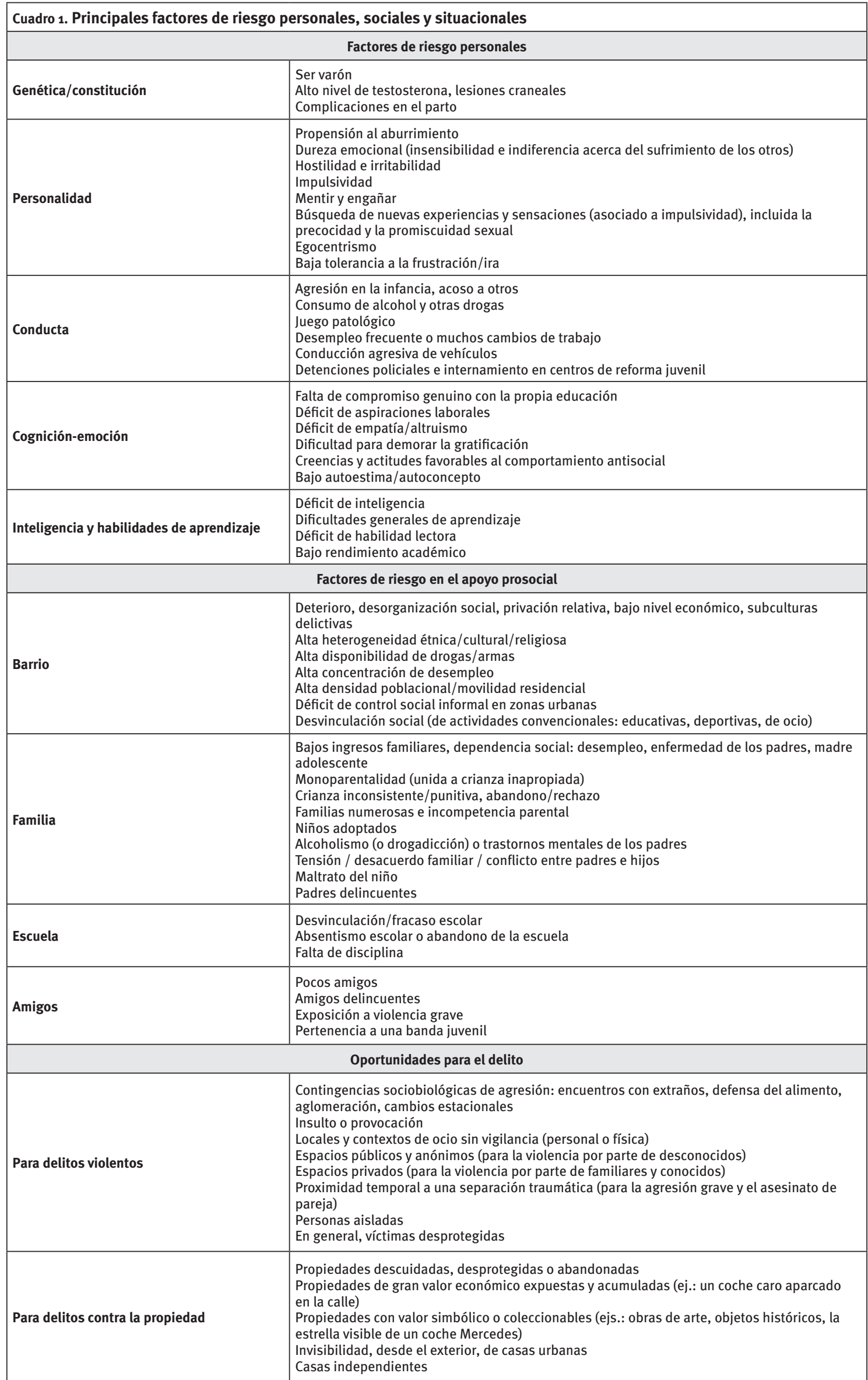

Fuente: Elaboración propia a partir de Redondo (2008a). 
son aquellos correlatos vinculados al pasado del individuo, o a otras características personales de difícil o nula modificación, como por ejemplo, hiperactividad, fracaso escolar o experiencias de maltrato en la infancia. En cambio, los factores de riesgo dinámicos, llamados necesidades criminogénicas, corresponden a aquellos elementos que sí podrían modificarse a través de una intervención, tales como cogniciones o actitudes delictivas, o hábitos antisociales. Las necesidades criminogénicas constituyen habitualmente los objetivos de los tratamientos con infractores y delincuentes adultos.

Desde un punto de vista rehabilitador, Andrews y Bonta (Andrews y Bonta, 2010; Andrews, 1996) han enfatizado cuatro grandes factores de riesgo a los que debería prestarse una atención preferente, por su especial relevancia en el desarrollo de la conducta delictiva y en su tratamiento:

1. Cogniciones antisociales del individuo, incluyendo actitudes, valores, racionalizaciones y estados emocionales favorables al comportamiento ilícito y antisocial.

2. Amigos antisociales de los menores, que claramente favorecerían la reincidencia delictiva.

3. Historia individual de comportamiento antisocial (edad de inicio, incidencia o frecuencia delictiva, grado de violencia utilizado, tipologías delictivas e internamientos previos).

4. Rasgos y factores de personalidad antisocial, tales como agresividad, egocentrismo, impulsividad, psicopatía, y déficits de autocontrol y de capacidad para la resolución de problemas interpersonales.

Según estos autores, a los anteriores riesgos deben añadirse también los tres siguientes (Andrews y Bonta, 2010):

5. Factores familiares (en la familia de origen) de influencia criminógena, tales como deficientes niveles de afecto, falta de cohesión familiar, pobre supervisión y crianza paternas (lo que incluye crianza punitiva), y abandono y abuso infantil.

6. Bajos niveles educativos y de formación laboral, y, especialmente, inestabilidad en el empleo.

7. Abuso de sustancias tóxicas (Gendreau, Little y Goggin, 1996).

\subsection{La transición desde la delincuencia juvenil a la adulta}

Como se ha visto, sólo un pequeño porcentaje de jóvenes son los que persisten y agravan su comportamiento criminal. Una de las explicaciones que se presentan ante dicha situación considera que el grado de motivación antisocial o delictiva que tengan los jóvenes va a determinar que algunos de ellos se conviertan en delincuentes crónicos o no. Dicho constructo ha sido detallado en el modelo del triple riesgo delictivo (TRD) [Redondo, 2008a]. Según Redondo, la motivación antisocial se definiría como la disposición individual para implicarse en actividades ilícitas y delictivas si se presentan oportunidades favorables para ello (la motivación antisocial también puede conllevar una búsqueda activa de oportunidades delictivas).

La motivación antisocial de un sujeto vendría definida por la confluencia en él de los factores de riesgo personales y sociales descritos anteriormente con detalle. Así, cuanto más fuerte sea la interacción en un mismo individuo de algunas de las características de riesgo personales y de algunos de los riesgos en el apoyo prosocial recibido, mayor probabilidad tendrá de presentar una elevada motivación antisocial.

Pero además, tal y como se deriva de la definición de este concepto en el modelo TRD, para el análisis del riesgo real de comportamiento delictivo también se requiere tomar en consideración las oportunidades a las que el individuo se ve expuesto. A partir de ello, se deriva el segundo constructo importante de este modelo, el de riesgo de conducta antisocial, que hace referencia a la probabilidad real de que un individuo realice un hecho delictivo concreto. A partir de lo anterior, podrían presentarse dos situaciones opuestas: 1) incluso en sujetos 'antisocialmente motivados', para delinquir se necesita que se presente una ocasión u oportunidad favorable para el delito, como pueda ser la presencia en la calle de un objeto deseado y desprotegido, o de una víctima vulnerable; pero, además, 2) para el caso de sujetos con nula o escasa 'motivación antisocial', la disponibilidad de evidentes oportunidades delictivas puede incrementar su riesgo infractor.

\subsubsection{Factores de riesgo y tratamiento}

Según la perspectiva teórica anterior, las tres grandes fuentes de riesgo aducidas requieren tres tipos distintos de intervenciones e iniciativas preventivas. Los factores de tipo personal, tales como impulsividad, pensamientos distorsionados o reacciones emocionales de ira, van a necesitar intervenciones educativas y de tratamiento directas con los individuos. Estas intervenciones son las que se aplican fundamentalmente en el marco de las medidas de justicia juvenil.

En cambio, los factores de riesgo sociales, tales como crianza paterna inapropiada, desvinculación escolar, posibles amigos delincuentes o barrios deteriorados, requerirán desarrollar en la sociedad, de modo paulatino, programas y mejoras sociales de amplio espectro. No obstante, los efectos preventivos de este tipo de intervenciones sobre la reducción de la delincuencia juvenil se obtendrán a medio y largo plazo, y afectarán a generaciones futuras de jóvenes (es decir, no a los actuales delincuentes juveniles). Pese a ello, este tipo de programas preventivos son 
absolutamente necesarios para lograr una reducción de la delincuencia a largo plazo.

Por último, las oportunidades delictivas podrían reducirse a través de medidas de prevención ambiental, como por ejemplo, aumentar los controles sociales informales, mejorar la iluminación de las calles o potenciar la visibilidad de los espacios. Sin embargo, dichos factores no pueden ser eliminados por completo, ya que muchos de ellos forman parte del propio bienestar y riqueza de la sociedad (Felson, 2006).

Como conclusión de todo lo expuesto en este epígrafe, no debería esperarse que las intervenciones educativas y los tratamientos que se aplican en el marco de la justicia juvenil acaben produciendo una eliminación total de la reincidencia delictiva. Más bien, lo razonable sería esperar que dichos tratamientos puedan disminuir parcialmente el riesgo delictivo futuro de los menores infractores.

\section{Programas de tratamiento $y$ rehabilitación: fundamentos y eficacia}

Los tratamientos con jóvenes infractores se dirigen a dotar a los sujetos de nuevas habilidades y competencias de comunicación e interacción humanas, a ofrecerles nuevas experiencias para el integración social (familiar, educativa, laboral) y a promover en ellos la inhibición de ciertas actitudes para las conductas infractoras (Israel y Hong, 2006; Palmer, 2003).

Según se ha visto, los tratamientos implican esencialmente modificar ciertos factores de riesgo dinámicos presentes en los infractores, que constituyen los objetivos fácticos de la intervención. De esta forma, el diseño y la aplicación de un tratamiento con menores infractores debe iniciarse con la evaluación de las necesidades y carencias relacionadas con su actividad delictiva. Para elegir el tratamiento más adecuado, debería, en todo caso, considerarse una intervención basada en un modelo teórico plausible del comportamiento delictivo y de su tratamiento. Después, se debería seleccionar, de entre las disponibles, aquella intervención que resulte más acorde a las necesidades de tratamiento que se han detectado, o en su defecto, adaptar una iniciativa ya existente, o bien diseñarla ex profeso. Para conseguir la máxima eficacia, el programa tiene que aplicarse de la manera más completa posible y según la temporalización prevista. Por último, es imprescindible evaluar la eficacia del tratamiento aplicado.

Tal y como se ha visto, uno de los requisitos esenciales para aplicar un tratamiento -que, a su vez, guarda estrecha relación con el éxito futuro de aquél- tiene que ver con la exigencia de que se fundamente en algún modelo teórico plausible sobre la conducta delictiva y la reincidencia. Son diversas las perspectivas sobre las que se han sustentado los programas de tratamiento con delincuentes, recogiéndose las más relevantes, de manera resumida, en el Cuadro 2.
En la actualidad, se aplican programas de tratamiento y rehabilitadores muy diversos por lo que se refiere a la base teórica sobre la que se fundamentan. Sin embargo, las perspectivas más empleadas y conocidas se corresponden a las mencionadas teoría del aprendizaje social y modelo de riesgonecesidades-responsividad (2010) [Cooke y Philip, 2001; Cullen y Gendreau, 2006; Howell, 2009; Lipsey, 2009; Ogloff y Davis, 2004; Redondo, 2008a], además del modelo cognitivo-conductual, que ha dado lugar al diseño de numerosas técnicas psicológicas de tratamiento de alta eficacia, aplicadas en muchos de los programas de rehabilitación con delincuentes (Redondo, 2008b).

En cuanto a la eficacia de los tratamientos, en general, se ha obtenido una eficacia entre moderada y baja. Concretamente, los metanálisis existentes obtienen un tamaño del efecto promediado de entre $r=0,07$ y $r=0,13$ Uolliffe y Farrington, 2009; Redondo, Sánchez-Meca y Garrido, 1999a, 1999b; Sánchez-Meca y Redondo, 2002; Latimer, 2001; Piquero, Jennings y Farrington, 2009; Lipsey, 1999a, 1999b). Es decir, las intervenciones aplicadas reducen entre el $7 \%$ y el $13 \%$ la reincidencia en los grupos tratados respecto a los grupos de control o no tratados. Pese a que la magnitud de dicha eficacia pueda resultar pequeña, es relevante en términos de reducción de posibles víctimas y agresores.

Los metaanálisis aquí revisados indican que la mayor eficacia terapéutica se da en las intervenciones fundamentadas en el modelo conductual y cognitivoconductual (con un tamaño del efecto promediado de $r=0,12$ ), así como, en aquellas que tienen como objetivo el desarrollo de habilidades de vida ( $r=$ $0,18)$. No obstante, con carácter general, las terapias cognitivo-conductuales podrían llegar a alcanzar una eficacia de en torno a $r=0,25$. También, las intervenciones basadas en comunidades terapéuticas con sistemas de organización estructurados y los programas de derivación de los jóvenes a la comunidad han demostrado una eficacia elevada $(r=0,12$ y $r=0,15$, respectivamente) [Redondo, 2008b; Redondo, Sánchez-Meca y Garrido, 2002a, 2002b]. En cambio, las intervenciones basadas en el modelo disuasorio, 0 de cariz meramente punitivo, como los llamados boot camps o pseudosistemas de entrenamiento militar, fundamentados en la disciplina y el castigo suelen mostrar baja o nula eficacia terapéutica $(r=0,00$ y $r=$ 0,01) [Cooke y Philip, 2001; Cullen y Gendreau, 2006].

\subsection{Intervenciones educativas desarrolladas con menores infractores en España}

Los servicios de justicia juvenil, encargados de la ejecución de las medidas con menores en las diferentes comunidades autónomas españolas, desarrollan numerosas actividades educativas y terapéuticas. Dichas actividades pretenden ayudar a los jóvenes y dotarles de las herramientas necesarias para que se produzca una mejora en sus déficits en habilidades, actitudes y pensamientos antisociales, o en control 


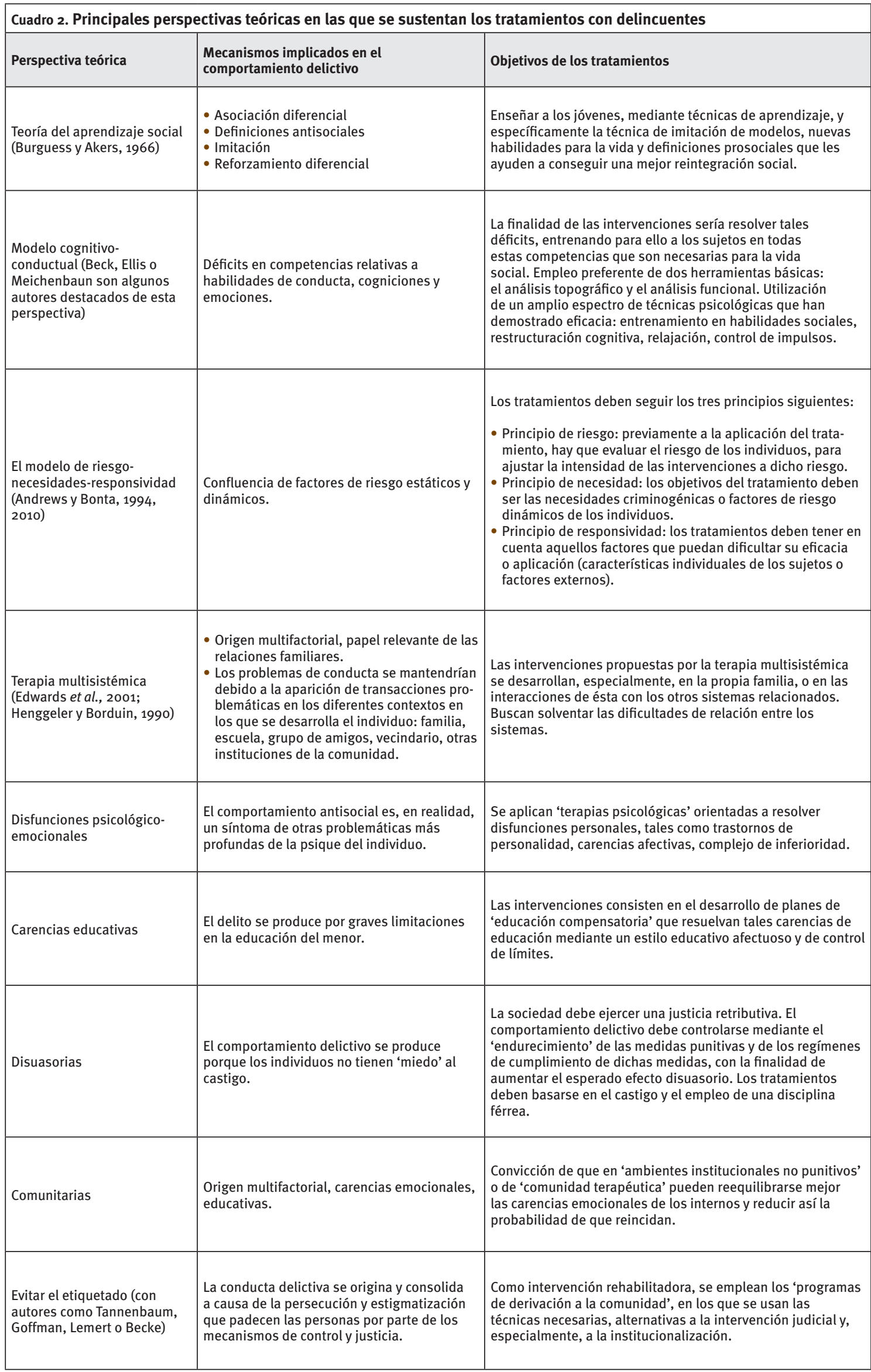

Fuente: Elaboración propia a partir de Gacono et al. (2001); Milan (2001); Morris y Braukmann (1987); Redondo (2006); y Redondo, SánchezMeca y Garrido (2002a, 2002b). 
de emociones, que, en definitiva, les ayuden a una mejor reintegración social.

A continuación, se describen brevemente las siete categorías en las que se agrupan las principales intervenciones aplicadas en España con menores:

- Actividades educativas y escolares, tales como cursos de alfabetización y educación reglada (primaria, secundaria), fomento de la lectura y escritura, o talleres de nuevas tecnologías. Mediante estas actividades, se pretende hacer frente al bajo rendimiento académico, el alto nivel de absentismo o el fracaso escolar de estos menores.

- Actividades prelaborales y laborales, dirigidas a aumentar las capacidades de los jóvenes para obtener y mantener un empleo, y así alcanzar un medio de subsistencia honrado. Algunas de estas actividades son talleres y cursos de formación ocupacional, prácticas profesionales, entrenamiento en habilidades específicas y programas de inserción laboral.

- Actividades de educación psicosocial, las cuales pretenden aminorar la fuerza de múltiples factores de riesgo mediante la enseñanza a los jóvenes de conocimientos y habilidades generales relevantes para la interacción social. Son ejemplo de este tipo de actuaciones los talleres para el desarrollo de las tareas de la casa o las habilidades de comunicación, la educación afectiva y sexual, la seguridad vial, la educación maternal o la autogeneración de un proyecto de vida.

- Intervenciones psicoterapéuticas y tratamientos. Estas actuaciones presentan un cariz más intenso y estructurado que las anteriores, aunque su objetivo también es disminuir el efecto de los múltiples factores de riesgo sobre el menor. Se trataría de programas y tratamientos específicos, para la prevención del maltrato familiar o la agresión sexual, el control de los impulsos, el entrenamiento en resolución de conflictos, o programas de gestión del riesgo de reincidencia.

- Intervenciones en salud y trastornos mentales, tales como numerosas actuaciones médicas y psiquiátricas, la gestión de las tarjetas sanitarias, la asistencia a especialistas, la atención a trastornos mentales y drogodependencias, además, de la prevención.

- Actividades de ocio y tiempo libre, encaminadas a enseñar a los jóvenes a planificar su tiempo libre de manera apropiada y prosocial. Con esta intención, se realizan distintos programas de entretenimiento, actividades culturales y deportivas, visitas a instalaciones comunitarias, juegos o lecturas.

- Intervenciones con los menores y sus familias, aplicadas para ayudar a mejorar la relación entre los menores y sus entornos familiares, así como para enseñarles patrones educativos equilibrados y consistentes. Son ejemplo de este tipo de actuaciones los programas de promoción de la implicación familiar en la educación de los jóve- nes, de mediación y resolución de conflictos en la familia, o la enseñanza de prácticas educativas efectivas.

\section{Conclusiones}

Hasta aquí se han repasado múltiples resultados de la investigación científica sobre carreras delictivas, los modelos de tratamiento con infractores juveniles más aplicados en la actualidad y las actuaciones realizadas en justicia juvenil en España.

De acuerdo con la información revisada, la delincuencia juvenil y adulta es un fenómeno complejo y multicausal, en el que diversos factores de riesgo y de protección se asocian a la probabilidad de comisión de un delito. Según se ha visto, los diversos correlatos de riesgo para el delito se han organizado en tres grandes fuentes: a) riesgos personales; $b$ ) riesgos en el apoyo prosocial recibido; y c) riesgos relativos a las situaciones y oportunidades favorecedoras de los delitos. Los riesgos a los que los tratamientos juveniles deben dirigirse son aquellos factores que propenden a aumentar la motivación antisocial de los sujetos, los cuales coinciden en buena medida con los denominados factores de riesgo dinámicos, o de necesidad criminogénica (correlatos más dúctiles al cambio que los denominados factores estáticos) [Israel y Hong, 2006; Polaschek y Reynolds, 2001]. No obstante, la delincuencia en un territorio no puede abordarse solamente mediante los tratamientos psicológicos, sino que la prevención de las infracciones juveniles requiere distintos planos de intervención, siendo el tratamiento sólo uno de ellos.

A grandes rasgos, un tratamiento o programa (de educación o rehabilitación) es uno de los medios técnicos de que se dispone en la actualidad para reducir el riesgo delictivo de los jóvenes infractores. Los programas de tratamiento que han mostrado mayor eficacia son aquellos que se fundamentan en perspectivas teóricas sólidas, que explican los mecanismos vinculados al comportamiento delictivo, así como los medios y técnicas que deberán emplearse para la rehabilitación de los jóvenes.

Según el modelo de riesgos-necesidadesresponsividad, anteriormente descrito, los tratamientos más efectivos son aquellos que, previa evaluación, se dirigen a las principales necesidades criminogénicas de los individuos. Es decir, a resolver y paliar aquellos factores de riesgo que se vinculan de modo más directo con la conducta delictiva de los sujetos. En este sentido, como ha podido observarse, los objetivos de los siete grupos de actuaciones desarrolladas con jóvenes infractores en España parecen incidir en correlatos de riesgo relevantes, muchos de ellos destacados ya por Andrews y Bonta (2010). Así, mediante las diversas actividades aplicadas con los menores, es posible reducir las limitaciones educativas, laborales, sociales, de salud, de ocio 
y familiares de muchos de ellos. Por ejemplo, el correlato de riesgo de la variable 'amigos antisociales', que se ha destacado en la investigación como uno de los predictores más influyentes sobre los jóvenes, podría verse amortiguado a través de algunas de las actuaciones que se desarrollan en el área de ocio y tiempo libre.

Éstas tenderían a poner en contacto a los menores con jóvenes que, en general, muestran un comportamiento prosocial, y sustraerles de otras relaciones de amistad más problemáticas.
Así, conforme a toda la evidencia empírica existente, como resultado de las intervenciones aplicadas con los jóvenes que ejecutan una medida judicial, sería esperable que se redujera la intensidad de su actividad delictiva. Tal reducción podría suponer una disminución de la frecuencia delictiva, de su intensidad o gravedad, o bien que se acortara en el tiempo la duración de su carrera criminal (Redondo, 2008b). Igualmente, dichos resultados podrían concretarse en una cierta disminución de las tasas de reincidencia de los menores tratados y una disminución de las tasas de delincuencia juvenil en España. 
ANDREWS, D. (1996): “Criminal recidivism is predictable and can be influenced: An update", Forum on Corrections Research, vol. 8, nํㅜ 3, págs. 42-44 [rhttp://www.csc-scc.gc.ca/text/pblct/forum/ e083/083m_e.pdf〉].

ANDREWS, D.; y BONTA, J. (2010): The Psychology of Criminal Conduct, $5^{\underline{a}}$ ed., Cincinnati, Anderson Publishing Co.

- (1994): The Psychology of Criminal Conduct, Cincinnati, Anderson Publishing Co.

BECHTEL, K.; LOWENKAMP, C. T.; y LATESSA, E. (2007): "Assessing the risk of re-offending for juvenile offenders using the youth level of service/case management inventory", Journal of Offender Rehabilitation, vol. 45, ํㅜㄹ, págs. 85-108.

BERGMAN, L. R. (2009): IDA: Individual Development and Adaptation [<http://www.oru.se/English/ Research/Research-projects/project/School-ofLaw-Psycology-and-Social-work/IDA-IndividualDevelopment-and-Adaptation/>].

BERGMAN, L. R.; y ANDERSHED, A. (2009): "Predictors and outcomes of persistent or age-limited registered criminal behavior: A 30-year longitudinal study of a Swedish urban population", Aggressive Behavior, vol. 35 , nํㅜ 2, págs. 164-178.

BURGESS, R.; y AKERS, R. A. (1966): “Differential association-reinforcement", Theory of Criminal Behavior. Social Problems, vol. 14, $\mathrm{n}^{\circ} \mathrm{2}$, págs. 128-147.

COOKE, D. J.; y PHILIP, L. (2001): "To treat or not to treat? An empirical perspective", en HOLLIN, C. R. (ed.), Offender Assessment and Treatment, Chichester, Wiley.
CULLEN, F. T.; y GENDREAU, P. (2006): "Evaluación de la rehabilitación correccional: política, práctica y perspectivas”, en BARBERET, R.; y BARQUÍN, J., Justicia penal siglo XXI: una selección de Criminal Justice 2000, Granada, Editorial Comares, págs. 275-348.

EDWARDS, D. L. et al. (2001): “A multilevel perspective on the implementation of multisystemic therapy (MST): attempting dissemination with fidelity", en BERNFELD, G. A.; FARRINGTON, D. P.; y LESCHIED, A. W., Offender rehabilitation in Practice: Implementing and Evaluating Effective Programs, Chichester, Wiley, págs. 97-120.

FARRINGTON, D. (ed.) (2008): Integrated Developmental and Life-course Theories of Offending, New Jersey, Transaction Publishers.

- (1992): "Criminal career research in the United Kingdom", British Journal of Criminology, vol. 32, n- 4 , págs. 521-536.

FARRINGTON, D.; TTOFI, M.; y COID, J. (2009): “Development of adolescence-limited, late-onset, and persistent offenders from age 8 to age 48 ", Aggressive Behavior, vol. 35, $\mathrm{n} \div$ 2, págs. 150-163.

FELSON, M. (2006): Crime and Nature, Thousand Oaks, Sage.

GACONO, C. et al. (2001): "Treating conduct disorder, antisocial, and psychopathic personalities", en ASHFORD, J. B.; SALES, B.; y REID W. (eds.), Treating Adult and Juvenile Offenders with Special Needs, Washington D. C., American Psychological Association, págs. 99-130.

GARRIDO, V.; STANGELAND, P.; y REDONDO, S. (2006): Principios de criminología, $3^{\underline{a}}$ ed., Valencia, Tirant lo Blanch. 
GENDREAU, P.; LITTLE, T.; y GOGGIN, C. (1996): “A metaanalysis of the predictors of adult offender recidivism: What works?", Criminology, vol. 34, nº 4, págs. 575-607.

HENGGELER, S. W. (2003): “Advantages and disadvantages of multisystemic therapy and other evidencebased practices for treating juvenile offenders", Journal of Forensic Psychology Practice, vol. 3, nํ- 4, págs. 53-59.

- (1989): Delinquency in Adolescence, Newbury Park, Sage.

HENGGELER, S. W.; y BORDUIN, C. M. (1990): Family Therapy and Beyond: A Multisystemic Approach to Treating the Behavior Problems of Children and Adolescents, Pacific Grove, Brooks/Cole.

HOWELL, J. C. (2009): Preventing and Reducing Juvenile Delinquency, Los Ángeles, Sage.

INSTITUTO NACIONAL DE ESTADÍSTICA (2012): Seguridad y justicia. Estadística de condenados: menores [<http://www.ine.es/jaxi/ menu.do?type $=$ pcaxis $\&$ path $=\% 2 \mathrm{Ft} 18 /$ p467\&file=inebase $\& L=0>$ ].

ISRAEL, M.; y HONG, W. (2006): "If 'something works' is the answer, what is the question? Supporting pluralist evaluation in community corrections in the United Kingdom", European Journal of Criminology, vol. 3, nํㅡ 2, págs. 181-200.

JOLLIFFE, D.; y FARRINGTON, D. (2009): “A systematic review of the relationship between childhood impulsiveness and later violence”, Personality, Personality Disorder and Violence: An Evidence Based Approach, Chichester, Wiley-Blackwell, págs. 41-61.

LATIMER, J. (2001): "A meta-analytic examination of youth delinquency, family treatment, and recidivism", Canadian Journal of Criminology, $\mathrm{n}-43$, págs. 237-253.

LEE, E.; BEAVER, K.; y WRIGHT, J. (2009): Handbook of Crime Correlates, Kidlington, Elsevier.

LIPSEY, M. W. (2009): “The primary factors that characterize effective interventions with juvenile offenders: A meta-analytic overview", Victims \& Offenders, vol. 4, nํㅡ 2, págs. 124-147.

- (1999a): "Can intervention rehabilitate serious delinquents?", Annals of the American Academy of Political \& Social Science, $\mathrm{n}{ }^{\circ}$ 564, págs. 142-166.

- (1999b): “Can rehabilitative programs reduce the recidivism of juvenile offenders? An inquiry into the effectiveness of practical programs", Virginia Journal of Social Policy \& the Law, vol. $6, \mathrm{n}^{\circ}$ 3, págs. 611-641.

LOEBER, R.; FARRINGTON, D. P.; y WASCHBUSCH, D. A. (1998): "Serious and violent juvenile offenders", en LOEBER, R.; y FARRINGTON D. P. (eds.), Serious and Violent Juvenile Offenders, Thousand Oaks, Sage, págs. 313-345.

LÖSEL, F. (2000): “¿Existe un tratamiento eficaz para la psicopatía?:¿qué sabemos y qué deberíamos saber?", en RAINE, A.; y SANMARTín, J., Violencia y psicopatía, Barcelona, Ariel, págs. 235-272.

MCMURRAN, M.; y HOLLIN, C. (1993): Young Offenders and Alcohol-Related Crime: A Practitioner's Guidebook, Oxford, John Wiley y Sons.
MILAN, M. A. (2001): "Behavioral approaches to correctional management and rehabilitation", en HOLLIN, C. R. (ed.), Offender Assessment and Treatment, Chichester, Wiley, págs. 139-154.

MOFFIT, T. (1993): “Adolescence-limited and life-coursepersistent antisocial behavior: A developmental taxonomy”, Psychological Review, vol. 100, nํㅜ 4, págs. 674-701.

MORRIS, E. K.; y BRAUKMANN, C. J. (1987): “The dimensions of applied behavior analysis for crime and delinquency", en MORRIS, E. K.; y BRAUKMANN, C. J. (eds.), Behavioral Approaches to Crime and Delinquency, Nueva York, Plenum Press.

NILSON, A.; y ESTRADA, F. (2009): “Criminality and lifechances. A longitudinal study of crime, childhood circumstances and living conditions up to age 48", serie Reports, $\mathrm{n}^{\circ}$ 3, Estocolmo, Stockholm University, Department of Criminology.

OGLOFF, J. R. P.; y DAVIS, M. R. (2004): "Advances in offender assessment and rehabilitation", Psychology, Crime \& Law, vol. 10, $\mathrm{n}-3$, págs. 229-242.

PALMER, E. (2003): Offending Behaviour: Moral Reasoning, Criminal Conduct and the Rehabilitation of Offenders, Cullompton, Willan Publishing.

PIQUERO, A. R.; JENNINGS, W. G.; y FARRINGTON, D. P. (2009): Effectiveness of Programs Designed to Improve Self-Control, Estocolmo, Brottsförebyggande rådet.

POLASCHEK, D. L. L.; y REYNOLDS, N. (2001): “Assessment and treatment: Violent offenders", en HOLLIN, C. R. (ed.), Offender Assessment and Treatment, Chichester, Wiley, págs. 416-431.

RECHEA, C. (2008): Conductas antisociales y delictivas de los jóvenes de España, Consejo General del Poder Judicial; Centro de Investigación en Criminología de la Universidad de CastillaLa Mancha [<http://www.uclm.es/centro/ criminologia/pdf/informes/16_2008.pdf)].

RECHEA, C. et al. (1995): La delincuencia juvenil en España: autoinforme de los jóvenes, Universidad de Castilla-La Mancha; Ministerio de Justicia e Interior [<http://www.uclm.es/centro/ criminologia/pdf/informes/o0_1995.pdf`].

REDONDO, S. (2008a): "Individuos, sociedades y oportunidades en la explicación y prevención del delito: Modelo del Triple Riesgo Delictivo (TRD)", Revista Española de Investigación Criminológica, art. 7, nㅇ 6 [shttp://www. criminologia.net/pdf/reic/ano6-2008/ a62008art7.pdf>].

- (2008b): Manual para el tratamiento psicológico de los delincuentes, Madrid, Pirámide.

- (2006): "Crime control through treatment of offenders", [conferencia plenaria], $6^{\text {th }}$ Annual Conference of the European Society of Criminology, Tübingen, 26-28 de agosto.

REDONDO, S.; y GARRIDO, V. (2013): Principios de criminología, Valencia, Tirant lo Blanch.

- (2001): Violencia y delincuencia juvenil, Mendoza, Ediciones Jurídicas Cuyo. 
REDONDO, S.; SÁNCHEZ-MECA J.; y GARRIDO, V. (2002a): "Los programas psicológicos con delincuentes y su efectividad: La situación europea”, Psicothema, vol. 14, suplemento, págs. 164-173.

- (2002b): "Crime treatment in Europe: A review of outcome studies", en MCGUIRE, J. (ed.), Offender Rehabilitation and Treatment: Effective Programmes and Policies to Reduce Re-offending, Sussex, Wiley, págs. 113-141.

- (1999a): "The influence of treatment programmes on the recidivism of juvenile and adult offenders: An European meta-analytic review", Psychology, Crime, \& Law, vol. 5, nํㅜ 3, págs. 251-278.

- (1999b): "Tratamiento de los delincuentes y reincidencia: una evaluación de la efectividad de los programas aplicados en Europa", Anuario de Psicología Jurídica, vol. 9, págs. 11-37.

REMSCHMIDT, H.; y WALTER, R. (2009): Kinderdelinquenz: Gesetzesverstösse Strafmündiger und ihre Folgen, Heidelberg, Springer.
SAMPSON, R.; y LAUB, J. (2005): "A life-course view of the development of crime", Annals of the American Academy of Political and Social Science, $\mathrm{n}-602$, págs. $12-45$.

SÁNCHEZ-MECA, J.; y REDONDO, S. (2002): “Metaanálisis de la eficacia de los programas de rehabilitación de delincuentes juveniles en Europa para la reducción de la reincidencia en el delito" [documento inédito], Departamento de Psicología Básica y Metodología de la Universidad de Murcia.

SARNECKI, J. (2009): “Delinquent networks. Youth cooffending”, en SCHNEIDER, H. J. (ed.), Internationales Handbuch der Kriminologie, vol. 2, Berlín, De Gruyter Recht.

- (1985): Predicting Social Maladjustment: Stockholm Boys Grown Up, Estocolmo, Brottsförebyggande rådet.

STENBERG, S. et al. (2007): "Stockholm Birth Cohort Study 1953-2003: A new tool for life-course studies", Scandinavian Journal of Public Health, vol. 35, no 1, págs. 104-110. 



\section{Normas de presentación de originales para Zerbitzuan}

- Zerbitzuan está abierta a la publicación de artículos sobre servicios y políticas sociales por parte de profesionales, investigadores, representantes institucionales y de todas aquellas personas interesadas en las políticas de bienestar social.

- Todos los artículos deberán ser inéditos y girar en torno a los ejes temáticos básicos de la revista (servicios sociales y políticas de bienestar social, participación social, inmigración, pobreza y exclusión social, discapacidad, atención a las personas mayores, infancia y menores en situación de desprotección, drogodependencias e intervención social en general). Una vez recibidos, los artículos serán sometidos a la consideración de los miembros del Consejo de Redacción, que decidirán sobre su publicación de acuerdo a criterios de calidad científica y oportunidad editorial.

- Una vez aceptados los artículos para su publicación, la dirección de la revista se reserva el derecho de modificar el título y epígrafes de los textos, así como de realizar las correcciones de estilo que se estimen convenientes, de acuerdo con las normas de estilo de la revista.

- Los artículos se enviarán en un documento de Word y por correo electrónico.

- Los artículos pueden redactarse tanto en euskera como en castellano, y serán publicados en el idioma en el que se reciba el original.

- Desde el punto de vista formal, se ruega atenerse a las siguientes pautas:

- La extensión del contenido de cada artículo será de entre 6.000 y 15.000 palabras, contando las posibles tablas, cuadros, gráficos y bibliografía. Se ruega adjuntar sólo las tablas y gráficos que se consideren imprescindibles.

- En la primera página, se hará constar el título del artículo, el nombre del autor o autores, su dirección y teléfono de contacto, así como su adscripción institucional. Se añadirá también un breve resumen o sumario del artículo (máximo 150 palabras) y entre cuatro y seis palabras clave que describan el contenido del texto. El resumen y las palabras clave irán en castellano y euskera.

- Las citas o notas irán a pie de página.

- Las referencias bibliográficas de los artículos seguirán las Normas ISO 690/1987.

La dirección y teléfono de contacto de la revista son los siguientes:

Zerbitzuan

SIIS Centro de Documentación y Estudios

SIIS Dokumentazio eta Ikerketa Zentrua

General Etxague 10 bajo

20003 Donostia-San Sebastián

Tel. 943423656

Fax 943293007

publicaciones@siis.net 


\section{핔|R|引|ㅍUAN}
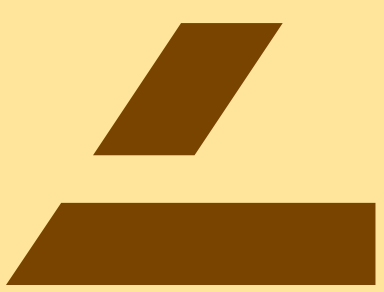

ABENDUA·DICIEMBRE

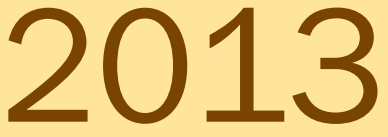

Pobreza infantil en Euskadi

Zaurgarritasun ibilbideetan balantzaka

Las prestaciones económicas de dependencia en Gipuzkoa: concurrencia, impacto y supervisión

La prestación económica de asistencia personal en el territorio histórico de Gipuzkoa

Estudio de la adopción y el abandono de modelos de gestión de la calidad en centros gerontológicos de la CAPV

Cuantificación y caracterización de la exclusión residencial en las capitales de la CAPV

El fin del sinhogarismo en Euskadi ¿mito o realidad?

El acompañamiento social personalizado como fórmula innovadora de respuesta a personas con trastorno mental y en exclusión social

Evaluar iniciativas de desarrollo comunitario desde la perspectiva de la innovación social. El caso de Gazteleku en el barrio de Rekalde (Bilbao)

La evolución del compromiso ético de los trabajadores sociales (1869-2013)
Objetivos y medios idóneos relativos a la dependencia funcional

Carreras delictivas juveniles y tratamiento

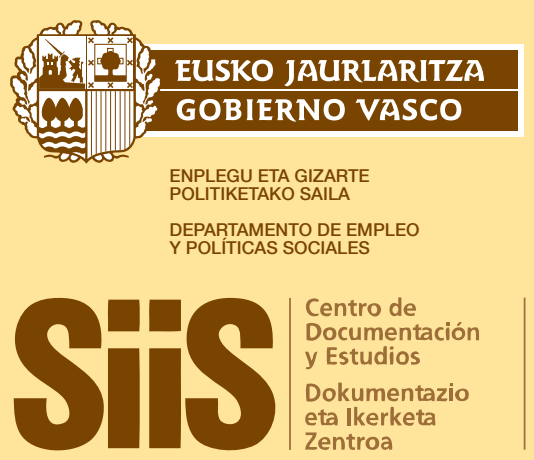

Fundación Eguia-Careaga Fundazioa 